\title{
QoS and QoE Aware Routing Protocol for Flying Ad-Hoc Surveillance Networks Using Fuzzy Inference Systems
}

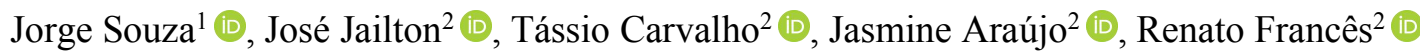 \\ ${ }^{I}$ Federal Rural University of Amazon (UFRA), Belém, Pará, Brazil, ZIP: 66.077-830 \\ ${ }^{2}$ Institute of Technology, Federal University of Pará (UFPA), Belém, Pará, Brazil, ZIP: 66.075-110 \\ jorge.souza@ufra.edu.br,jjj@ufpa.br,tassio@ufpa.br,jasmine@ufpa.br,rfrances@ufpa.br
}

\begin{abstract}
The Flying Ad-hoc Network (FANET) networks have often operated in regions that are difficult to reach and have no fixed infrastructure. The use of devices such as Unmanned Aerial Vehicles (UAV) enable aerial networks to be created that are extremely fast, although there are no specific routing protocols for this type of network that can make communication more efficient among these devices. For this reason, this paper sets out a specific routing protocol for FANET networks that is designed for the discovery of routes among UAV devices. Evidence of the benefits of this strategy has been obtained through simulation by the Network Simulator version 2.
\end{abstract}

Index Terms - FANET, Flight Autonomy, Quality of Experience, Routing Protocol, Fuzzy System.

\section{INTRODUCTION}

The traditional concept of Ad-Hoc Networks has been adapted to the creation of a new concept called the Flying Ad-Hoc Network (FANET). In this new context, FANETs consist of Unmanned Aerial Vehicles (UAVs) for the creation of networks in local areas where it is difficult to access terrestrial UAVs, particularly after natural disasters. UAVs are responsible for monitoring a certain area by capturing images that are sent to a base station on the ground [1].

One of the main challenges in FANETs is how to position the UAVs in a suitable way to enable them to monitor the region. The positioning of the UAVs is of strategic importance for the network as a means of establishing a connection among them. Moreover, owing to their mobility (UAV's ability to move in different directions and speeds), the devices might either draw closer or keep a distance from one another and hence risk impairing communication among the UAVs. The degree of mobility is also an important factor since the UAVs might either fly too slowly or quickly, which would also jeopardize the connection [2].

Another important factor is the flight autonomy of the UAVs, since most of the devices have an average flight time of 30 minutes [3], [4]. A UAV with a low flight autonomy will have to stop being Brazilian Microwave and Optoelectronics Society-SBMO received 22 July 2019; for review 23 July 2019; accepted 22 Oct 2019 Brazilian Society of Electromagnetism-SBMag 
part of the network because it requires a topology restructuring, which may affect the connection among UAVs. There is a need to determine which devices have a low flight autonomy and predict the restructuring of the network topology so as to reduce its impact on the connection.

UAV monitors a particular area by capturing images in real time, so it is important to assess the quality of those imagens. It can be performed by using Quality of Experience (QoE) metrics. The term Quality of Experience is related to the evaluation of multimedia applications from the point of view of user perception [5]. The term emerged to fill the "gaps" left by the traditional evaluation made by Quality of Service metrics such as flow, jitter and delay, which shows the impact of applications from a network standpoint. The Quality of Service (QoS) metrics do not reflect the user experience of a video and do not allow to qualify the quality of the video received.

As already mentioned, the mobility and flight autonomy are factors that require changes in the network topology. In light of this, a routing protocol is needed that is capable of interacting with the network changes. There is currently no specific routing protocol for FANET and the traditional AdHoc routing protocols such as Ad-hoc On-Demand Distance Vector (AODV) and Optimized Link State Routing (OLSR) do not perform satisfactorily [6].

Owing to the challenges raised by FANET, this paper sets out a specific routing protocol for FANET using a Fuzzy System to find the best communication pathway between the UAVs. The routing protocol will discover the route that has the best connection and will also be active for a longer period of time.

This paper is structured as follows: The related works are discussed in Section 2. Section 3 outlines the FANET applications and Section 4 describes the proposed routing protocol in detail. The results obtained from the simulation are shown in Section 5. Section 6 summarizes the conclusion and makes suggestions for future work.

\section{RELATED WORK}

This section examines the related work on the routing protocol Flying Ad-hoc Networks (FANET's). The related works were analyzed to demonstrate that our routing protocol proposed has greater and significant benefits than those discussed in other papers available in the literature. In [7], the authors analyzed the Quality of Service (QoS) metrics for the AODV, OLSR and HWMP routing protocols in Flying Ad-Hoc Networks (FANETs) with the aid of an NS-3 simulation tool. The paper used the Gauss-Markov Mobility Model and calculated the throughput metrics and corresponding overheads for the protocols. However, it only compares the above-mentioned protocols and does not recommend any improvement for them. In [8], the authors devise a new mechanism for data routing based on localization in GPS-denied or GPS-challenged areas. This mechanism relies on a weighted centroid localization technique, where the position of unknown UAV nodes is calculated by means of fuzzy 
logic. This paper does not take into account that changes in topology require new packet routing or the flight autonomy of each drone. In [9], the authors investigated a routing protocol called P-OLSR (OLSR predictive), which is an extension of the traditional OLSR protocol. The authors compared the P-OLSR with OLSR by means of QoS metrics. However, although the paper put forward a new routing protocol, it did not compare it with the other routing protocols and failed to evaluate it with the QoE metrics. In [10], the authors recommended an energy-efficiency algorithm for drones that included the parameters of the communication channel. This system reduced energy consumption, but even though there were a number of changes in the topology, the network was unable to identify new routes in an effective way and thus maintain the required level of quality. In [11], the authors carried out a survey of the routing protocols for VANET and FANET. Their paper discusses the use of optimization techniques (Particle Swarm Optimization, Ant Colony Optimization and Bee Colony Optimization) to improve the routing performance but did not employ flight autonomy as a parameter or take QoE metrics into account. In [12], the authors discuss the use of hop-by-hop communication between the drones as a means of increasing the network coverage area. However, the paper does not discuss to what extent the changes in the topology can have an effect on the quality of transmission, while also failing to address the need for an efficient routing protocol.

The authors in [7]-[12] examine the techniques that are used in FANET, but none of them set out a routing protocol that provides an effective response to the changes in the network topology. These papers do not address flight autonomy of the drones either and do not use a computer intelligence system for decision making.

The proposed Routing Protocol, unlike related work presented in the literature, addresses the needs and challenges of a FANET. Table I shows related works that broadly addresses FANET routing and its solutions. This paper proposes an alternative approach to existing FANET's routing protocols by using Fuzzy Systems. Our proposed routing protocol was validated by QoE metrics. 
TABLE I. RELATED WORK

\begin{tabular}{|c|c|c|c|}
\hline Proposal & $\begin{array}{c}\text { Flight } \\
\text { Autonomy }\end{array}$ & Decision Strategy & Proposal Focus \\
\hline [4] & No & $\begin{array}{c}\text { To use Gauss- } \\
\text { Markov Mobility } \\
\text { Model }\end{array}$ & $\begin{array}{l}\text { Evaluate traditional } \\
\text { routing protocol in } \\
\text { FANETs }\end{array}$ \\
\hline [5] & No & $\begin{array}{l}\text { Mechanism for data } \\
\text { routing based on } \\
\text { localization }\end{array}$ & $\begin{array}{l}\text { The position for } \\
\text { UAV's device }\end{array}$ \\
\hline [6] & No & $\begin{array}{c}\text { Extension of OLSR } \\
\text { protocol called P- } \\
\text { OLSR }\end{array}$ & $\begin{array}{l}\text { Compare P-OLSR } \\
\text { with OLSR }\end{array}$ \\
\hline [7] & No & $\begin{array}{l}\text { Energy -efficiency } \\
\text { algorithm. }\end{array}$ & $\begin{array}{l}\text { To reduce the } \\
\text { energy } \\
\text { consumption }\end{array}$ \\
\hline [8] & No & $\begin{array}{l}\text { Survey of the routing } \\
\text { protocols }\end{array}$ & $\begin{array}{l}\text { Improve the } \\
\text { routing } \\
\text { performance }\end{array}$ \\
\hline [9] & No & $\begin{array}{l}\text { Discuss the hop-by- } \\
\text { hop communication }\end{array}$ & $\begin{array}{l}\text { Number of UAV's } \\
\text { necessary }\end{array}$ \\
\hline $\begin{array}{l}\text { Current } \\
\text { Proposal }\end{array}$ & Yes & $\begin{array}{l}\text { Routing Protocol } \\
\text { with Fuzzy Logic } \\
\text { System }\end{array}$ & $\begin{array}{l}\text { Selecting the best } \\
\text { routing } \\
\text { communication } \\
\text { considering Flight } \\
\text { Autonomy with } \\
\text { mobility and RSSI }\end{array}$ \\
\hline
\end{tabular}

\section{Flying AD-Hoc NeTwork (FANET)}

There is now a type of network configuration that has evolved from the concept of Mobile Ad-Hoc Networks (MANET) and Vehicular Ad-Hoc Networks (VANET) as well as from Wireless Sensor Networks. This type of configuration has led to an increased number of resources and is constantly growing, especially in the production of new devices and systems that are complex and able to move and fly autonomously.

In FANET, the devices are generally referred to as Unmanned Aerial Vehicles (UAVs). The use of UAVs has created new ways of operating innovative applications, by introducing a new type of network paradigm known as FANETs.

The networks differ from their traditional predecessors (MANETs in this case) as a result of their degree of mobility, greater connectivity and an increase in application areas, etc. In this respect, FANETs can generalize and extrapolate the topologies from 2D to 3D through a free-motion scheme, owing to the ability of the drones to fly independently in three-dimensional space. This new context has attracted researchers and industry as well as providing a driving-force for real-life applications.

FANET networks are generally used to provide connectivity to hard-to-reach places in regions where there have been natural disasters, or even for military applications. After a catastrophic event 
(such as an earthquake, hurricane, tsunami, dam breach, etc.), traditional network infrastructures can suffer damage and be subject to automatic shutdowns. However, through a FANET configuration, they could be employed to restore and provide sufficient connection and communication to the network in isolated areas. In addition, UAVs could be equipped with cameras and other types of sensors and devices to provide a constant aerial view and thus help rescue crews and firefighters to save lives.

In extensive coverage areas, it may be impracticable to establish direct communication from the UAVs to the base station on the ground at certain times. However, this problem can be overcome through hop-by-hop communication, which requires the use of a routing protocol to discover the best route/path from the original source to the final destination [13].

\section{A. Problem Statement and Major Contributions}

One of the main problems in this type of network is to determine and maintain the routes, since the mobility of the UAVs can cause changes in the topology. For this reason, the main focus of this paper is to set out a specific routing protocol for FANET networks that can accomplish the task more efficiently [14].

The routing protocols are responsible for finding, establishing, and maintaining routes between two nodes that wish to communicate with each other. These protocols must generate the minimum possible overhead and the bandwidth consumed by them must also be small.

A routing protocol that targets FANET networks is more complex than fixed network protocols; this is due to several features of these networks such as their dynamic topology algorithm, mutual interference, restricted power and the limited resources available in the UAVs.

In FANET networks, if one UAV is not close enough to another to carry out the communication, it will have to make use of routing information to choose the best path. The communication among UAVs that is beyond the reach of transmission, is made in multiple hops through the collaboration of intermediary nodes; that is, the scope is not restricted to the radius of action of each device individually, but to the sum of the radius of action of all of the devices (Fig. 1).

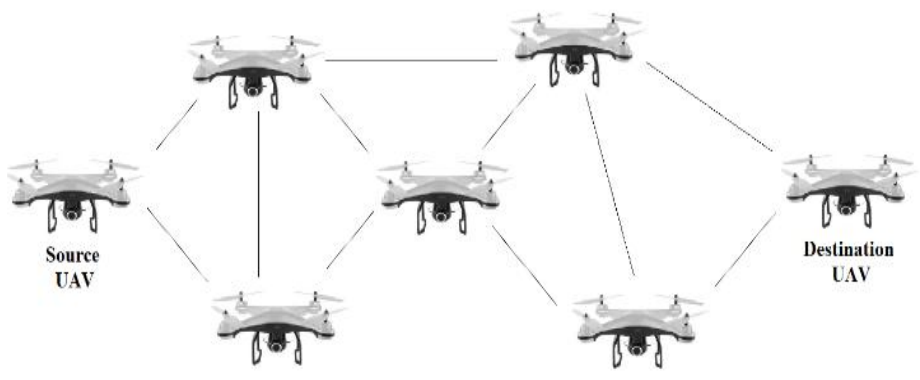

Fig. 1. Hop-by-Hop Communication in FANET. 
The mobility of UAVs and their spatial arrangement are also very important for determining the communication routes. As a result of the movement, these routes are usually re-arranged so that the interconnection between the UAVs can be continued. For this reason, the routing must be carried out dynamically by increasing the autonomy of the UAVs and reducing the delay in data delivery between a source node and a destination node [15].

Another main contribution of this paper is the adoption of a new communication network model used to provide connectivity in regions that are difficult to reach on land (especially regions after natural disasters). FANETs are easily established, as they are easily moved to a new region.

The frequent updating of the control information can ensure more accurate information; however, there is a need for a greater use of energy, since this reduces the autonomy of the nodes. For this reason, this paper proposes a specific routing protocol for FANET networks where the Received Signal Strength Indicator (RSSI), mobility level and in particular the flight autonomy of each UAV, are employed as decision-making metrics to ensure Quality of Service and Quality of Experience for the network.

This paper also proposes, as a contribution, the use of a Fuzzy System for the implementation of the routing protocol, with a set of inputs composed of information collected, in real time, from the network itself. The information collected are: flight autonomy, mobility level and RSSI. Based on such information it is possible to establish communication routes that will remain active for a longer period of time. The objective is to choose routes with high flight autonomy (longer service life), low mobility (less changes in the topology) and with better RSSI (better communication for data transmission).

FANETs are commonly employed to monitor regions by using sensors to capture images and/or videos Therefore, it is very important that the quality of the streamed video can be assessed using QoE metrics to ensure that, in fact, good data communication reflects a good user experience. Therefore, this article performs a cross-layer evaluation involving the network and application layers to verify it.

\section{FUZZY ROUTING PROTOCOL SYSTEM}

Fuzzy System allows the use of variables that are dynamic and imprecise, which makes it ideal for scenarios where values are often changing. As the values change, a new solution is found for the network. This paper examines three metrics for input: Mobility Level, Flight Autonomy and RSSI (These three entries are shared between UAV's by routing protocol signaling packets).

In order to define the RSSI, mobility and flight autonomy intervals, the values imposed by the technology [3] were used as upper limit and, through simulation, adjustments were made in such a 
way that the FANET's objectives could be achieved to a greater or lesser degree.

The RSSI is an indicator of the signal quality received by the user. The better the RSSI, better is the communication between two drones. The worse the RSSI, means the drones are at a distance from each other, the worse will be the communication. Three sets were also defined for the RSSI (dBi): Low (interval [-125.1 to -102.1$]$, medium (interval [-111.1 to -63.1] and high (when the RSSI is lower than -71.1).

The mobility is an important metric that can affect the transmission quality, since it can indicate how fast two or more drones are either drawing apart or approaching each other. Three bands were defined for mobility: Low mobility (interval [0 to 5]), medium mobility (interval [4 to 13] and high mobility (when the speed is higher than $11 \mathrm{~m} / \mathrm{s}$ ).

The flight autonomy is another important metric that has been used to measure how long the drone will monitor the region. The longer the flight time, the longer the route will be available for communication. The metric was also divided into three categories: low (range 0 - 10 minutes), medium (range 10 - 20 minutes) and high (over 20 minutes).

The Fuzzy System will have one output variable that can be classified as follows: TERRIBLE route, REGULAR route, GOOD route and EXCELLENT route. When the drone detects a new route, it will provide the input variables collected for the Fuzzy System, which after the Fuzzyfication process, it will indicate the quality level of the detected route. Fuzzyfication is the mapping of input variables to a set of ranges that will be parsed by a fuzzy rule base and defuzzyfication is the mapping of the inference machine output to a fuzzy inference value of a set of linguistic variables [16]. The Fuzzy System will discover the route that has the best level of quality.

In this paper, the Gaussian fuzzifier was used due to its ability to reduce noise in the input variables [17]. The output inference value is analyzed to identify to which set it belongs. After 100 iterations of the simulation process, the value 0.6 was found to be good or excellent for the route. In certain situations, an inference value can be part of two sets at the same time (such as 0.55), in which case, the metric that has the most relevance will be decisive in identifying the output set (Fig. 2).

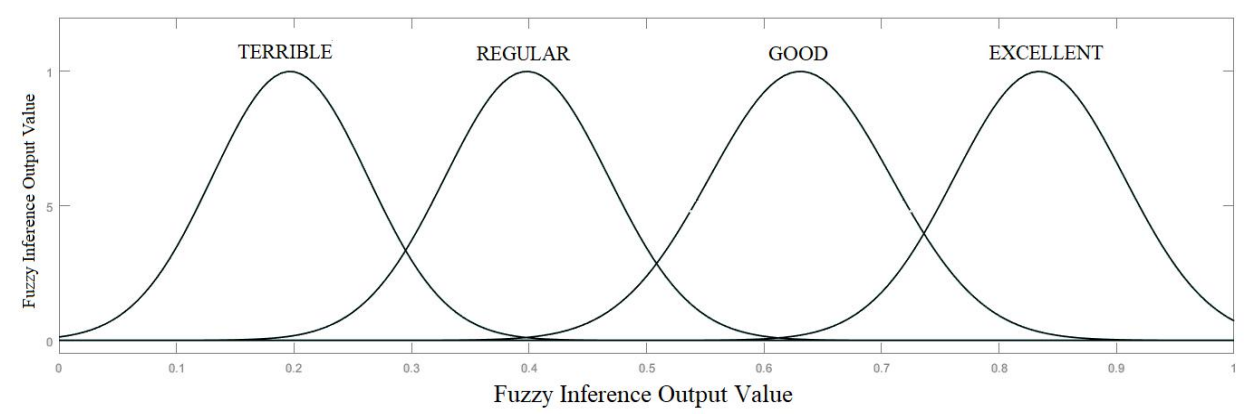

Fig. 2. Output of the Fuzzy System. 
The ideal communication will be between drones that have a high flight autonomy, high RSSI and low mobility. This type of setting allows the route to be longer for the transmission. However, these conditions are not always possible, and in this case, the Fuzzy System will try to find out which route is closest to it.

The following table shows the main rules of the Fuzzy System which has an excellent or good route. In these cases, the drone will select the route that will remain active for longer and hence achieve the best performance. The other situations that are not shown in the Table, correspond to TERRIBLE or REGULAR route that will rarely be chosen by the Fuzzy System, and then only in cases where GOOD or EXCELLENT routes are not available (Table II).

TABLE II. A FuZzy RULE BASEd SySTEM

\begin{tabular}{cccc}
\hline Mobility & $\begin{array}{c}\text { Flight } \\
\text { Autonomy }\end{array}$ & RSSI & Output \\
\hline LOW & MEDIUM & MEDIUM & GOOD \\
LOW & HIGH & MEDIUM & GOOD \\
MEDIUM & MEDIUM & MEDIUM & GOOD \\
MEDIUM & HIGH & MEDIUM & GOOD \\
LOW & MEDIUM & HIGH & EXCELENT \\
LOW & HIGH & HIGH & EXCELENT \\
MEDIUM & HIGH & HIGH & EXCELENT \\
\hline
\end{tabular}

The final decision is made in accordance with the inference value (resulting from the output of the Fuzzy System), and the highest inference value will be chosen for decision- making. During the implementation of the Fuzzy System, it was observed that the inference values equal to, or greater than, 0.6 represent the best routes and hence are very likely to be chosen. In the following graph, the yellow area represents the most likely routes to be chosen, since they correspond to the routes in which the drones have high flight autonomy, low mobility and high RSSI. In general, the part of the graph shaded in green, corresponds to the drones with medium mobility, medium RSSI and medium flight autonomy, and in this type of situation, there is little chance of the drone being chosen as a communication route. The blue region of the graph represents a drone with high mobility, low RSSI and low flight autonomy, and in this case the drone will not be chosen as the communication route (Fig. 3).

In general, for a UAV to select a transmission route, it transmits to the Fuzzy Inference machine the RSSI, mobility, and flight autonomy range information, received from neighboring UAVs. The Fuzzy Inference Machine, through the Fuzzy rule base, will generate an output value to evaluate the quality level of the route. The route with the highest rating and the highest outbound inference value will be chosen by the UAV to initiate transmission to the destination UAV (Fig. 4). 


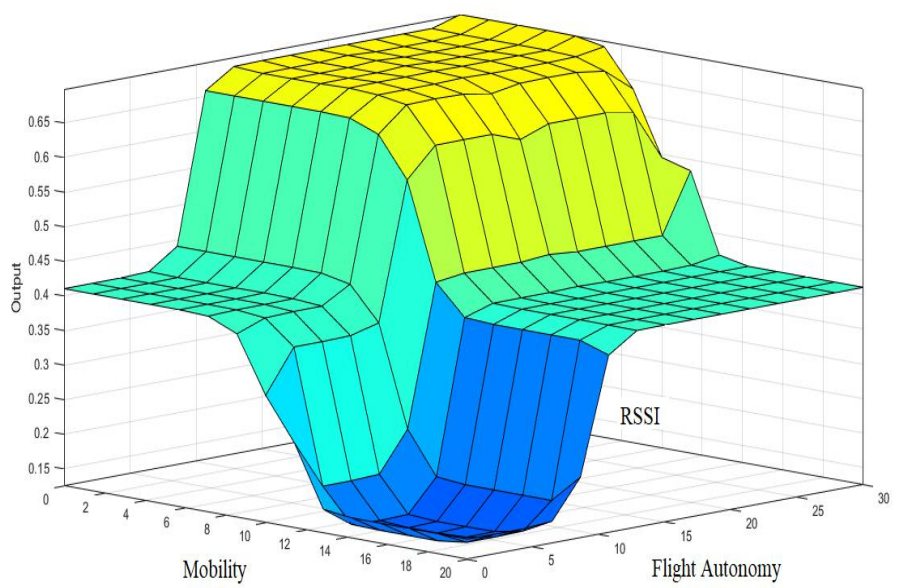

Fig. 3. Output of the Fuzzy System.

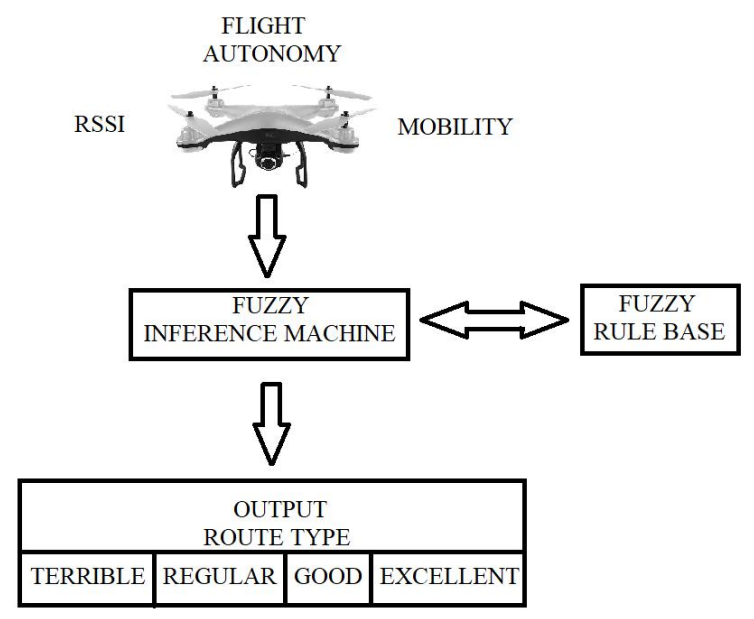

Fig. 4. Schematic Representation of the Proposal

\section{RESULTS}

This section shows the benefits of the proposal, as well as the results which were obtained through a simulation conducted in Network Simulator version 2 (NS-2). The simulations evaluated the performance of the AODV (reactive protocol), OLSR (proactive protocol) and Fuzzy-Based Adaptive protocols with a multimedia application. The video used in the simulations was Highway which has a resolution of $352 \times 288$ and 2000 frames.

The simulator does not have support for three-dimensional simulations (or other simulators), and as a result, it is assumed that the drones are flying at a similar height and also within sight of communication. In the simulation process, the propagation model adopted was Shadowing, due its realistic representation of signal attenuation than Free Space Model and Two Ray Ground (the 
propagation models available in simulator) [18]. The simulation was carried out by 10 drones with random mobility (Random Way Point) with a speed ranging from $2 \mathrm{~m} / \mathrm{s}$ to $20 \mathrm{~m} / \mathrm{s}$ in an area of $200 \mathrm{~m} \mathrm{x}$ $200 \mathrm{~m}$. The simulation parameters are given in Table III.

TABLE III. NS-2 CONFIGURATION PARAMETERS

\begin{tabular}{cc}
\hline Parameter & Value \\
\hline Drones & 10 \\
Access Technology & IEEE $802.11 \mathrm{~g}$ \\
Propagation Model & Shadowing \\
Mobility type & Random Waypoint \\
Query & Droptail \\
Number of Simulations & 100 \\
Confidence Interval & $95 \%$ \\
Frequency & $2.4 \mathrm{GHz}$ \\
Area & $200 \mathrm{~m} \mathrm{x} 200 \mathrm{~m}$ \\
\hline
\end{tabular}

Due to random movements as well as the speed of the UAVs, they may fly in the same or in opposite directions. This aspect may make them approach or fly far from each other either faster or slower. These changes in the topology require a fast response from the routing protocols and if this does not occur, the network performance will be impaired.

The throughput graph shows the best performance of the Fuzzy Adaptive Protocol compared with other protocols. Owing to the topology variations, AODV and OLSR protocols were unable to update the routes quickly and efficiently, and as a result, none of the protocols was able to keep the route active. For this reason, both protocols break the transmission during part of the simulation. The Fuzzy Adaptive Protocol was able to update the route quickly and efficiently, while at the same time always keeping the route active and preventing any break in the connection (Fig. 5).

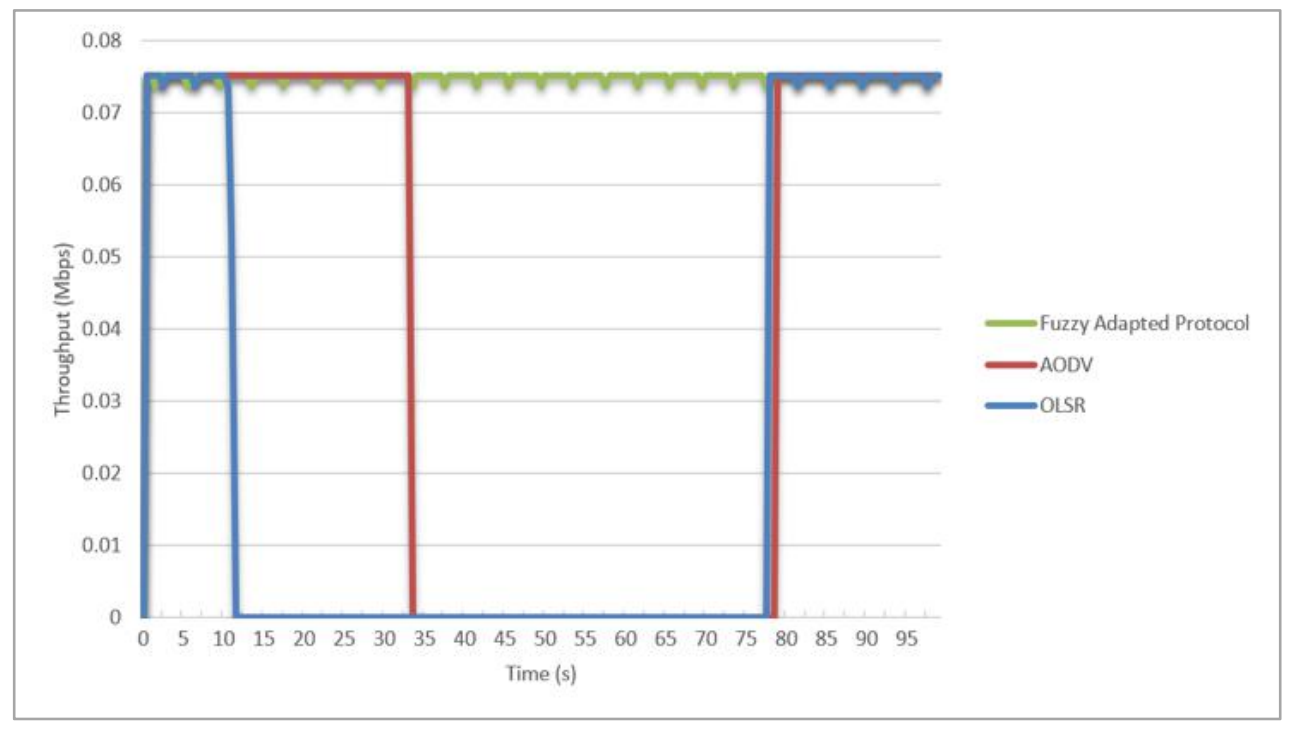

Fig. 5. Throughput over time. 
The best performance of the proposed protocol can be found when the throughput averages are compared. The OLSR protocol had an average throughput rate of $0.26 \mathrm{Mbps}$, the AODV protocol had an average throughput rate of $0.45 \mathrm{Mbps}$, while the Adapted Fuzzy protocol had an average throughput rate of $0.72 \mathrm{Mbps}$. The adapted fuzzy protocol had a $176 \%$ gain over OLSR and a $60 \%$ gain over AODV. The following graph shows the average throughput rates for each protocol (Fig. 6).

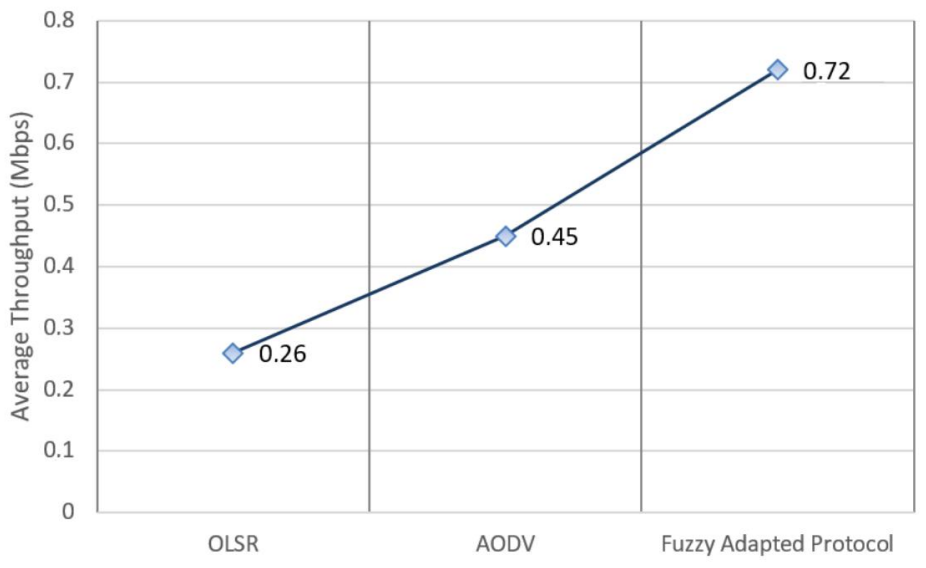

Fig. 6. Average Throughput.

The throughput directly interferes with the number of frames received by the user and hence affects the quality of the video. The interruption of the throughput during a certain period of time leads to a reduction in the number of frames that are normally received. As mentioned earlier, the video had 2000 frames. Figure 7 shows that the OLSR protocol received 400 frames, the AODV protocol received 1400 frames and the Fuzzy Adaptive protocol received 2000 frames.

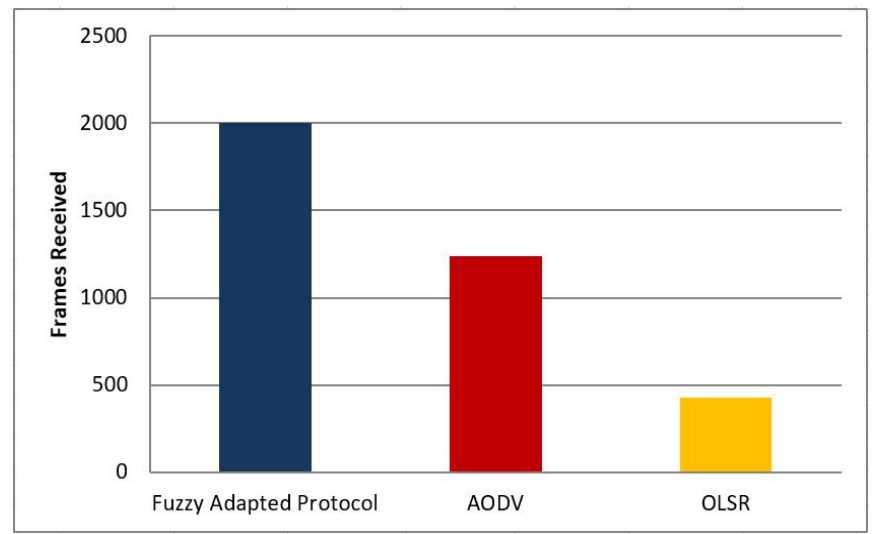

Fig. 7. Number of Frames Received.

This paper also evaluated the benefits of the proposal through the Quality of Experience metrics. The QoE metrics compare the video received by the user with the original video. The three main QoE metrics are: Peak Signal-to-Noise Ratio (PSNR), Structural Similarity (SSIM) and Video Quality Metric (VQM). The PSNR evaluates the signal-noise of the video with regard to the following 
features: brightness, noise and color. In the following graph the OLSR protocol had a PSNR average of $18 \mathrm{~dB}$ (considered to be a bad video), the AODV protocol had an average of $27 \mathrm{~dB}$ ( a regular video) and the Fuzzy Adaptive protocol had an average of $41 \mathrm{~dB}$ (and thus is thought to be an excellent video) (Fig.8).

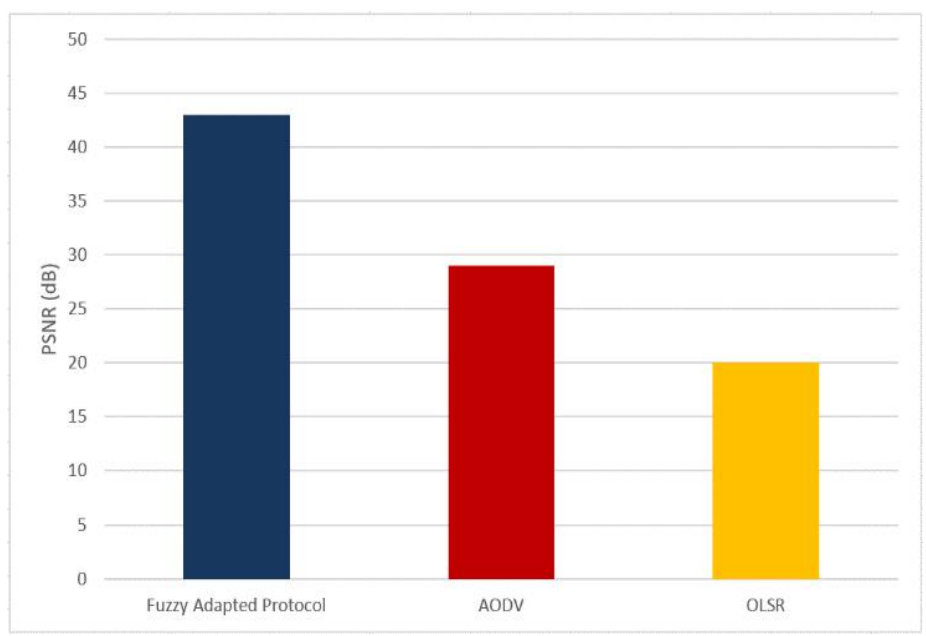

Fig. 8. PSNR values.

When the protocols that use the SSIM metric were compared, it was found that the Fuzzy Adaptive protocol also had a better performance. The SSIM also compares received video with the original video in terms of the following features: brightness, color and contrast. The range of the SSIM metric is on a scale of 0 to 1 - the value close to 1 means a better video quality. The OLSR protocol had an average SSIM value of 0.43 , the AODV protocol had an average of 0.61 and the Fuzzy Adaptive protocol had an average of 0.92 (Fig. 9).

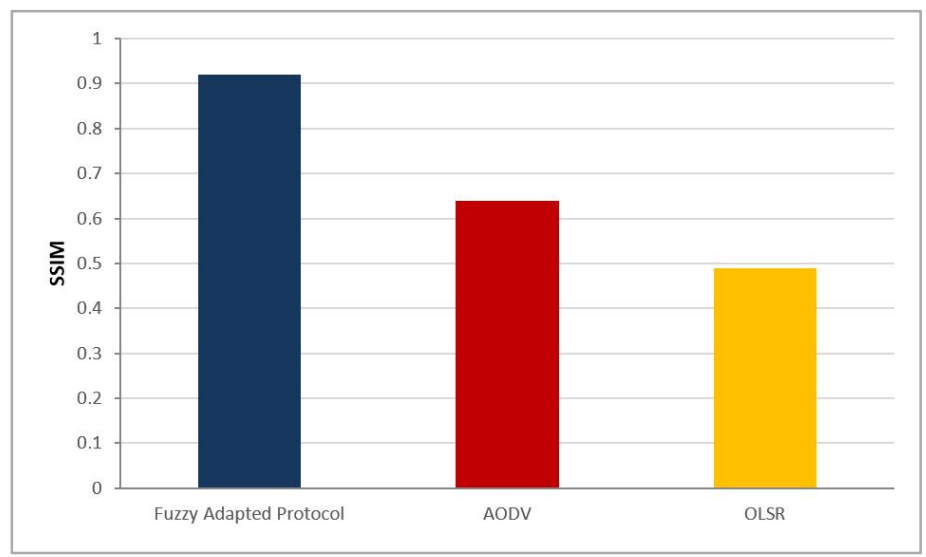

Fig. 9. SSIM values.

The VQM metric measures the following features: color, brightness, intensity, and frame distortion. It was also used to evaluate the video received by the user compared with the original video. The range of the VQM metric is on a scale of 0 to 5 ; in this case the value closer to 0 means a better video quality, that is, the distortion is less than in the original video. The OLSR protocol had a VQM 
average of 4.1 (i.e. a low-quality video), the AODV protocol had an average of 3.3 (a regular video) and the Fuzzy Adaptive protocol had an average of 1.4 (a video of excellent quality). Graph X displays the VQM values for each protocol (Fig. 10).

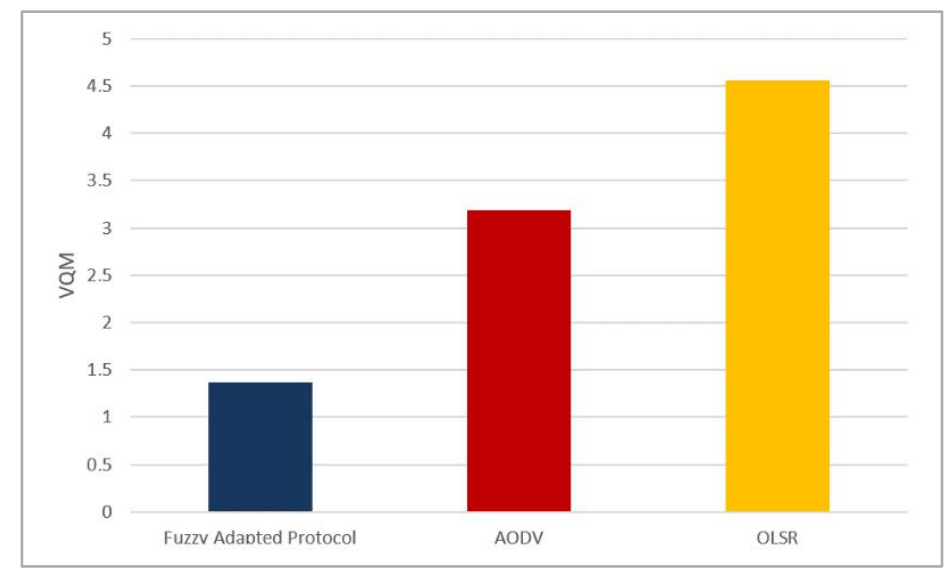

Fig. 10. VQM values.

In video simulation, the protocol achieved a $127 \%$ gain over the OLSR protocol and a $51 \%$ gain over the AODV protocol. In addition to evaluating the video received through the Quality of Experience metrics, this paper has also made a visual evaluation of the received video frames using the MSU Video Quality Measurement Tool Software [19]. On the basis of the visual evaluation, it can be stated that the Fuzzy Adaptive System had a better performance than the other protocols. Figure 11 makes it possible to analyze the frame of the video transmitted through the OLSR Protocol. In this case, the frame is quite distorted, and there are several defects since it is a frame of low quality.

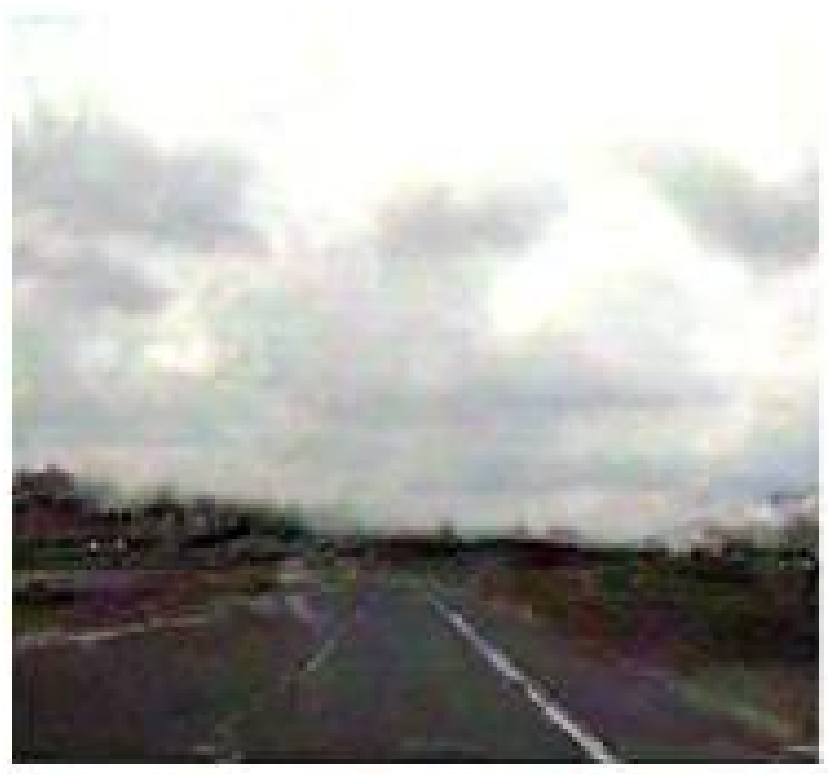

Fig. 11. Frame received by OLSR.

With regard to frame of the video transmitted through the AODV Protocol, it is clear that there is an improvement in quality when compared with the previous frame. However, the frame still has minor 
distortions and defects. Although it is not a poor-quality frame, it cannot be concluded that it is an excellent frame. The video has thus been classified as having a regular quality (Fig. 12).

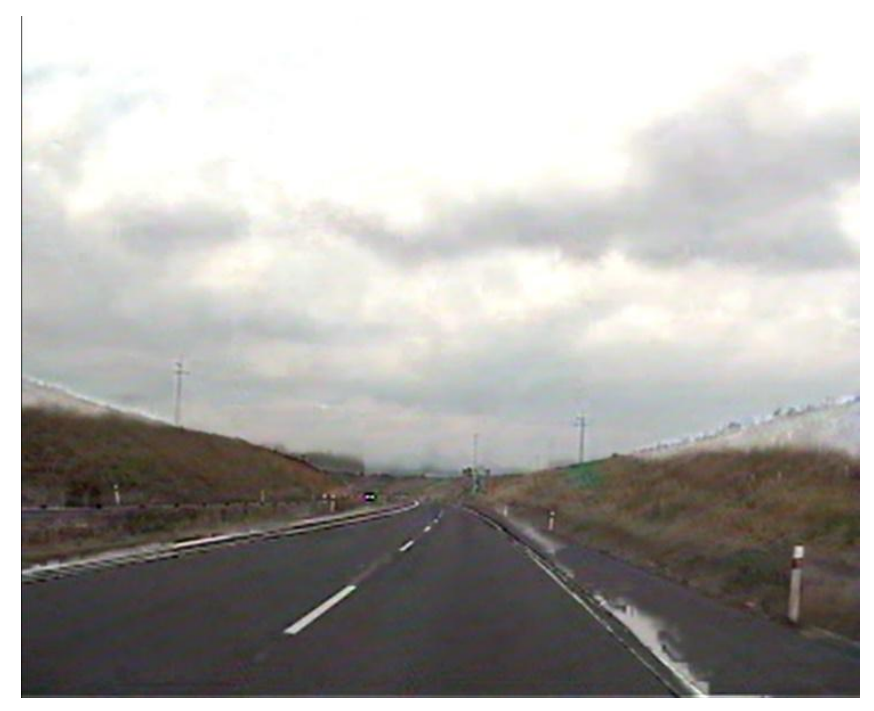

Fig. 12. Frame received by AODV.

When the frame of the video transmitted with the Fuzzy Adaptive Protocol is analyzed, it can be seen that there is a great improvement of quality with regard to the other two protocols. In Figure 13 below, as the frame has no distortions or defects like the previous frames, the video transmitted with the proposed protocol is rated as being of excellent quality.

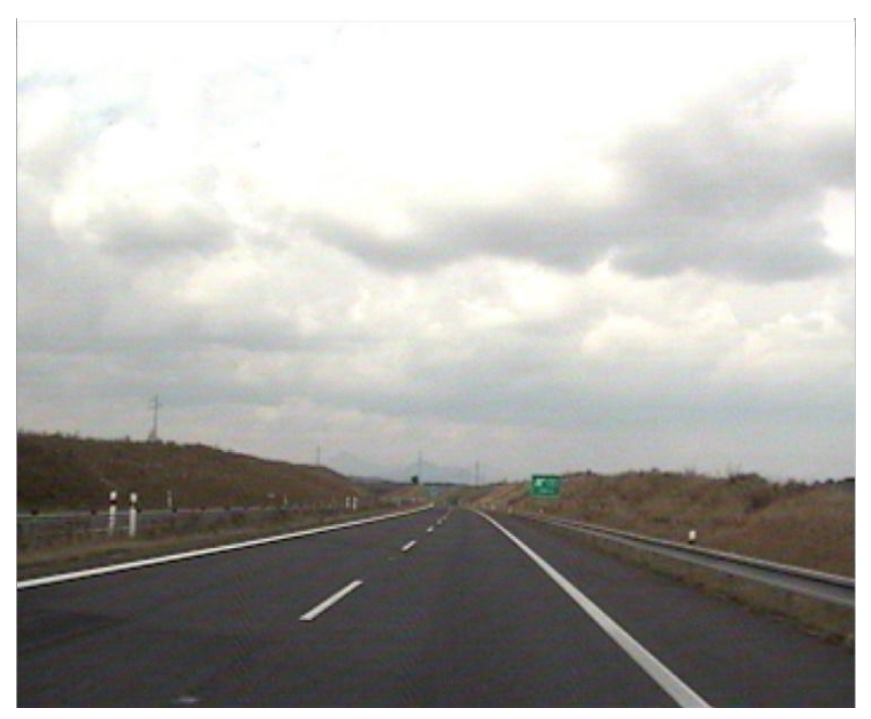

Fig. 13. Frame received by the Fuzzy System Routing Protocol.

\section{CONCLUSION}

The aerial networks have been the object of a good deal of research owing to the ease of creating and implementing them. Although the FANETs networks provide a number of benefits (as mentioned earlier) there are also a number of challenging tasks that have to be carried out.

One of these is finding the most efficient communication system among the UAVs by means of a 
routing protocol. For this reason, this paper has recommended a specific routing protocol for FANET networks which involves using a Fuzzy System to improve the route discovery process, while taking into account the RSSI, mobility level and in particular, the flight autonomy.

The proposed routing protocol was compared with the traditional Ad-Hoc routing protocols: AODV and OLSR. The comparison was carried out by means of traditional QoS and QoE metrics and the proposed routing protocol achieved a better performance (around 35\%) than the other two routing protocols referred to.

In future work the authors intend to employ new artificial intelligence techniques, and include new parameters for decision making, as well as making use of new wireless technologies and new propagation models.

\section{REFERENCES}

[1] E. Cruz, A Comprehensive Survey in Towards to Future FANETs.: IEEE Latin America Transactions, 2018.

[2] O. Burdakov, P. Doherty, K. Holmberg, Relay Positioning for Unmanned Aerial Vehicle Surveillance.: The International Journal of Robotics Research, 2018.

[3] Phantom 4 User Manual V 1.6. (2017). Accessed: Jan. 10, 2019. [Online]. Available: https://dl.djicdn.com/downloads/phantom_4/20170706/Phantom_4_User_Manual_v1.6.pdf

[4] Mavic 2 Pro Zoom User Manual V 2.0. (2019). Accessed: Jan. 10, 2019. [Online]. Available: https://dl.djicdn.com/downloads/Mavic_2/20190417/Mavic_2_Pro_Zoom_User_Manual_v2.0_en.pdf

[5] S. Winkler, "Perceptual video quality metrics - a review," in Digital Video Image Quality and Perceptual Coding, CRC Press, 2005.

[6] Z. Zheng, A. Sangaiah, T. Wang, "Adaptive Communication Protocols in Flying Ad Hoc Network," IEEE Communications Magazine, vol. 56, no. 1, Jan. 2018.

[7] D. S. Vasiliev, D. S. Meitis, A. Abilov, "Simulation-Based Comparison of AODV, OLSR and HWMP Protocols for Flying Ad Hoc Networks," in Balandin S., Andreev S., Koucheryavy Y. (eds) Internet of Things, Smart Spaces, and Next Generation Networks and System, 2014, pp. 245-252, doi: 10.1007/978-3-319-10353-2_21.

[8] F. Khelifi, A. Bradai, K. Singh and M. Atri, "Localization and Energy-Efficient Data Routing for Unmanned Aerial Vehicles: Fuzzy-Logic-Based Approach," IEEE Communications Magazine, vol. 56, no. 4, pp. 129-133, Apr. 2018.

[9] A. Nayyar, "Flying Adhoc Network (FANETs): Simulation Based Performance Comparison of Routing Protocols: AODV, DSDV, DSR, OLSR, AOMDV and HWMP," in Int. Conf. on Advances in Big Data, Computing and Data Communication Systems (icABCD), Durban, 2018, pp. 1-9.

[10] P. Kaur, A. Singh, "Nature-Inspired Optimization Techniques in VANETs and FANETs: A Survey," in Bhattacharyya S., Chaki N., Konar D., Chakraborty U., Singh C. (eds) Advanced Computational and Communication Paradigms. Advances in Intelligent Systems and Computing, 2018, pp. 651-663, doi: 10.1007/978-981-10-8237-5_63.

[11] G. A. Litvinov, A. V. Leonov and D. A. Korneev, "Applying Static Mobility Model in Relaying Network Organization in Mini-UAVs Based FANET," in Systems of Signal Synchronization, Generating and Processing in Telecommunications (SYNCHROINFO), Minsk, 2018, pp. 1-7, doi: 10.1109/SYNCHROINFO.2018.8456951.

[12] O. S. Oubbati, A. Lakas, F. Zhou, M Günes and M. B. Yagoubi, "A survey on position-based routing protocols for Flying Ad hoc Networks (FANETs)," in Vehicular Communications, 2017, pp. 29-56, doi: 10.1016/j.vehcom.2017.10.003.

[13] W. Zafar and B. Muhammad Khan, "Flying Ad-Hoc Networks: Technological and Social Implications," in IEEE Technology and Society Magazine, vol. 35, no. 2, pp. 67-74, Jun. 2016.

[14] S. Rosati, K. Krużelecki, G. Heitz, D. Floreano and B. Rimoldi, "Dynamic Routing for Flying Ad Hoc Networks," in IEEE Transactions on Vehicular Technology, vol. 65, no. 3, pp. 1690-1700, March 2016.

[15] B. Yang, M. Liu and Z. Li, "Rendezvous on the Fly: Efficient Neighbor Discovery for Autonomous UAVs," in IEEE Journal on Selected Areas in Communications, vol. 36, no. 9, pp. 2032-2044, Sept. 2018.

[16] L. Wang, A Course in Fuzzy Systems and Control. 1st ed. Prentice Hall, 1996.

[17] Z. Ju and H. Liu, "Fuzzy Gaussian Mixture Models," in Pattern Recognition, vol. 45, no. 3, Mar. 2012, pp. 1146-1158, doi: 10.1016/j.patcog.2011.08.028.

[18] The ns Manual (formerly ns Notes and Documentation). (2011). Accessed: Mar. 3, 2015. [Online]. Available: https://www.isi.edu/nsnam/ns/doc/ns_doc.pdf.

[19] MSU Quality Measurement Tool. Accessed: Fev. 10, 2016. [Online]. Avalaible: https://www.compression.ru/video/quality_measure/vqmt_download.html. 